\title{
Tyrosine Kinase Inhibitor-Related Hepatotoxicity in Patients with Advanced Lung Adenocarcinoma: A Real-World Retrospective Study
}

This article was published in the following Dove Press journal: Cancer Management and Research

\author{
Jie Qian $\mathbb{D}^{1,2, *}$ \\ Xueyan Zhang ${ }^{2, *}$ \\ Bo Zhang ${ }^{2}$ \\ Bo Yan $^{2}$ \\ Lin Wang ${ }^{3}$ \\ Ping $\mathrm{Gu}^{2}$ \\ Weimin Wang ${ }^{2}$ \\ Huimin Wang ${ }^{2}$ \\ Baohui $\mathrm{Han}^{2}$ \\ 'Department of Emergency Medicine, \\ Shanghai Chest Hospital, Shanghai Jiao \\ Tong University, Shanghai, People's \\ Republic of China; ${ }^{2}$ Department of \\ Pulmonary Medicine, Shanghai Chest \\ Hospital, Shanghai Jiao Tong University, \\ Shanghai, People's Republic of China; \\ ${ }^{3}$ Department of Laboratory Medicine, \\ Shanghai Chest Hospital, Shanghai Jiao \\ Tong University, Shanghai, People's \\ Republic of China \\ *These authors contributed equally to \\ this work
}

\begin{abstract}
Purpose: Hepatic injury is a common side effect following tyrosine kinase inhibitor (TKI) therapy and our understanding usually comes from clinical trials. In this retrospective study, we aimed to investigate the characteristics, risk factors and regimen-related differences of epidermal growth factor receptor (EGFR)-TKI-related hepatic toxicity in patients with advanced lung adenocarcinoma (LAD).
\end{abstract}

Patients and Methods: Liver function tests were documented in 424 patients admitted into the Shanghai Chest Hospital between January 2014 and December 2016 with advanced (IIIB/ IV) LAD who received first-line gefitinib, erlotinib or icotinib. Hepatotoxicity was graded according to the Common Terminology Criteria for Adverse Events (CTCAE), version 4.0. The clinical spectrum and onset time of hepatic injury were evaluated. The risk factors of hepatic dysfunction were determined using a logistic regression analysis

Results: A total of 87 (20.5\%) patients experienced hepatotoxicity and 5.7\% were of grade 3/4 liver dysfunction. The median onset time of hepatotoxicity was 7 weeks. Presence of hepatitis virus (HR: 2.593, 95\% CI: 1.090-6.170, $\mathrm{P}=0.031$ ) and pretreatment liver impairment (HR: 3.460 , 95\% CI: $1.746-6.855, \mathrm{P}<0.001)$ were risk factors associated with increased risk of hepatotoxicity. Gefitinib (HR: 1.872, 95\% CI: 1.028-3.412, P=0.040) and erlotinib (HR: $3.578,95 \%$ CI: $1.683-7.609, \mathrm{P}=0.001$ ) had increased risk of hepatotoxicity compared to icotinib. Conclusion: The different toxic profile of EGFR-TKIs should be taken into account in the choice of treatment based on the patients' comorbidity.

Keywords: lung adenocarcinoma, hepatotoxicity, tyrosine kinase inhibitor, gefitinib, erlotinib, icotinib

\section{Introduction}

Lung adenocarcinoma (LAD) accounts for the majority of lung cancer which is one of the leading causes of cancer-associated mortality worldwide. ${ }^{1}$ The development of epidermal growth factor receptor (EGFR) tyrosine kinase inhibitors (TKIs) dramatically improves the prognosis of LAD patients harboring EGFR sensitive mutations. However, TKI-related liver injury is commonly seen. ${ }^{2}$ It took place in $12-70 \%$ of patients depending on different treatment and study populations. ${ }^{3-7}$ While mild hepatotoxicity usually diminished with prompt intervention, severe liver dysfunction may result in treatment delay or suspension. The current understanding of EGFR-TKI-related hepatotoxicity, however, mostly comes from clinical trials with stringent selection criteria. The real-world safety properties, the risk factors and agent-related difference of hepatotoxicity have not been largely studied.
Correspondence: Baohui Han Department of Pulmonary Medicine, Shanghai Chest Hospital, Shanghai Jiao Tong University, 24I ${ }^{\#}$ West Huaihai Road, Shanghai 230030, People's Republic of China

Tel +862162821990 ext. 142 I

Fax +862132260806

Email18930858216@I63.com 
In this retrospective study, we reviewed records of patients receiving gefitinib, erlotinib and icotinib as the first-line treatment for advanced LAD. Characteristics, risk factors and regimen-related differences of hepatotoxicity were investigated for a deeper understanding of TKIrelated hepatotoxicity.

\section{Patients and Methods}

\section{Patients}

Medical history of all patients admitted into the Shanghai Chest Hospital, China between January 2014 and December 2016 were reviewed. The inclusion criteria for this study were: 1) age greater than 18 years; 2) pathologically confirmed as LAD; 3) advanced stage (IIIB or IV); 4) EGFR mutated and receiving first-line TKIs (gefitinib, erlotinib, or icotinib) with complete laboratory data. 5) observation time after TKI initiation $\geq 2$ months. Patients receiving concurrent chemotherapy with TKIs were excluded. The enrollment process was carried out on consecutive patients and included all patients meeting the criteria. Among total of 2704 newly diagnosed stage IIIB/IV lung adenocarcinoma patients who were admitted into the hospital during the period, 593 EGFR mutated patients received fist-line TKIs. A total of 424 patients were included in the final analysis after excluding those with incomplete laboratory data $(n=62)$, TKIs other than gefitinib, erlotinib or icotinib $(\mathrm{n}=41)$, TKIs administration $<2$ months $(\mathrm{n}=52)$ and concurrent therapy $(\mathrm{n}=14)$. The study was approved by the hospital's ethic committee and was performed in accordance with the ethical standards of the Declaration of Helsinki. Informed consent was waived due to the retrospective nature of the study and the analysis used anonymous clinical data.

\section{Data Collection}

Baseline characteristics including age, sex, smoking history, drinking history, stage, Eastern cooperative oncology group (ECOG) performance score (PS), pretreatment liver function, whether or not having liver metastases was recorded. Hepatitis B and C virus serology were performed for all patients at the baseline visit. Patients with positive hepatitis B virus surface antigen ( $\mathrm{HBsAg}$ ) and hepatitis $\mathrm{C}$ virus antibody (HCV-Ab) were recorded. Drinking for a minimum of 6 months with an alcohol consumption $>50 \mathrm{~g} /$ day was defined as having a drinking history.

\section{Laboratory and Hepatotoxicity Assessment}

Liver function was examined at baseline visit, at least biweekly for the first 2 months after TKIs therapy and every 1-2 months afterwards. Parameters including aspartate aminotransferase (AST), alanine aminotransferase (ALT), and total bilirubin (TBil) were examined and results were reported as ULN values. Hepatotoxicity was graded according to the Common Terminology Criteria for Adverse Events (CTCAE), version 4.0. The onset time of hepatotoxicity was defined as the interval from the date of starting TKIs to the time of hepatotoxicity detected. Duration of hepatotoxicity was defined as the interval between the detection of abnormal liver function and the time liver function returned to normal.

\section{Statistical Analysis}

Baseline characteristics were quantified by applying descriptive statistics. The association between variables and hepatic dysfunction during treatment were evaluated using a logistic regression analysis. All statistical analyses were performed using SPSS 22.0 software (IBM, Armonk, NY, USA). A p-value of less than 0.05 was considered statistically significant.

\section{Results}

A total of 424 patients receiving EGFR-TKI were included. The overall study population was 185 (43.6\%) male, with a median (IQR) age of 60 (53-67) years. Most patients (78.8\%) were in stage IV. Patients with ECOG PS 2 accounted for $9.4 \%$. The number of patients with pretreatment liver impairment was $45(10.6 \%)$. A total of 25 patients showed the presence of hepatitis virus, among whom 23 had positive HBsAg, 1 had positive HCV-Ab and 1 had both positive HBsAg and HCV-Ab. Patients' clinical characteristics are summarized in Table 1.

\section{The Frequency, Mode, Onset and Duration Time of Hepatotoxicity}

A total of 87 (20.5\%) patients experienced hepatotoxicity after TKI administration. Mild liver dysfunction (grade 1/ 2) accounted for the majority $(n=82,94.3 \%)$ while 5 (5.7\%) patients were diagnosed with $\geq$ grade 3 hepatotoxicity.

The majority of hepatic toxicity were presented as an elevation of ALT/AST and a small proportion had increased TBil levels (Figure 1). 
Table I Patient Demographics and Baseline Characteristics

\begin{tabular}{|c|c|}
\hline Variable & All Patients $(n=424), n(\%)$ \\
\hline Age, median (IQR) & $60(53-67)$ \\
\hline \multicolumn{2}{|l|}{ Age } \\
\hline$<70$ years & $355(83.7 \%)$ \\
\hline$\geq 70$ years & $69(16.3 \%)$ \\
\hline \multicolumn{2}{|l|}{ Gender } \\
\hline Male & I85 (43.6\%) \\
\hline Female & $239(56.4 \%)$ \\
\hline \multicolumn{2}{|l|}{ Smoking Status } \\
\hline Never & $313(73.8 \%)$ \\
\hline Ever & III (26.2\%) \\
\hline \multicolumn{2}{|l|}{ Drinking History } \\
\hline No & $416(98.1 \%)$ \\
\hline Yes & $8(1.9 \%)$ \\
\hline \multicolumn{2}{|l|}{$\mathrm{HBsAg}$ or HCV-Ab } \\
\hline Absent & $399(94.1 \%)$ \\
\hline Present & $25(5.9 \%)$ \\
\hline \multicolumn{2}{|l|}{ Pretreatment Liver Impairment } \\
\hline No & 379 (89.4\%) \\
\hline Yes & $45(10.6 \%)$ \\
\hline \multicolumn{2}{|l|}{ ECOG PS } \\
\hline $0 \sim 1$ & $384(90.6 \%)$ \\
\hline 2 & $40(9.4 \%)$ \\
\hline \multicolumn{2}{|l|}{ Stage } \\
\hline IIIB & $39(9.2 \%)$ \\
\hline IV & $334(78.8 \%)$ \\
\hline Recurrent & $51(12.0 \%)$ \\
\hline \multicolumn{2}{|l|}{ Liver Metastasis } \\
\hline No & 405 (95.5\%) \\
\hline Yes & $19(4.5 \%)$ \\
\hline \multicolumn{2}{|l|}{ TKI Regimen } \\
\hline Gefitinib & $215(50.7 \%)$ \\
\hline Erlotinib & $57(13.4 \%)$ \\
\hline Icotinib & $152(35.9 \%)$ \\
\hline
\end{tabular}

Abbreviations: $\mathrm{HBsAg}$, hepatitis $B$ virus surface antigen; $\mathrm{HCV}$ - $\mathrm{Ab}$, hepatitis $\mathrm{C}$ virus antibody; ECOG PS, Eastern Cooperative Oncology Group performance score.

The median (IQR) onset time of hepatotoxicity was 7 (4-10) weeks with a range from 1 to 96 weeks. For patients with severe hepatotoxicity ( $\geq$ grade 3 ), the median duration of liver dysfunction was 6 weeks (range: 4-15 weeks) (Figure 2).

\section{Risk Factors for Hepatotoxicity and Different Profiles Among TKIs}

The multivariate analysis showed that presence of hepatitis virus (HR: 2.593, 95\% CI: $1.090-6.170, \mathrm{P}=0.031$ ) and pretreatment liver impairment (HR: 3.460, 95\% CI: 1.7466.855, $\mathrm{P}<0.001)$ were two risk factors associated with increased risk of hepatotoxicity. Age, gender, smoking and drinking history, ECOG PS, stage and the presence of liver metastasis were not significantly associated with the occurrence of liver injury (Table 2).

\section{Regimen-Related Differences of Hepatotoxicity}

The difference in hepatotoxicity among gefitinib, erlotinib and icotinib was compared through multivariate regression analysis by incorporating all other clinical characteristics.

The results showed that there was no significant difference in hepatotoxicity between gefitinib and erlotinib (HR: 1.693, 95\% CI: 0.874-3.285, $\mathrm{P}=0.119)$. However, both gefitinib (HR: 1.872, 95\% CI: $1.028-3.412, \mathrm{P}=0.040)$ and erlotinib (HR: 3.578, 95\% CI: 1.683-7.609, $\mathrm{P}=0.001$ ) produced more events of hepatotoxicity compared to icotinib (Table 3)

\section{Discussion}

The current study investigated the nature, the risk factors and regimen-related differences for hepatic toxicity in advanced LAD patients receiving first-line EGFR-TKI therapy. Our findings confirmed that most hepatotoxic events were mild and occurred within the first 2 months. Patients with pretreatment liver impairment and the presence of the hepatitis virus were more susceptible to liver impairment. A Chinese homegrown EGFR-TKI-icotinib seemed to be superior to gefitinib or erlotinib when the liver issue was concerned.

EGFR-TKI-induced liver injury is commonly seen clinically. Single clinical trials on lung cancer reported a variable incidence ranging from $12 \%$ to $70 \%$, with $1-18 \%$ of cases presenting with $\geq 3$ grade hepatotoxicity. ${ }^{3-7}$ The heterogeneity of the studied population may explain the various incidences of liver dysfunction in trials. In this retrospective study, we evaluated all patients including who would normally be excluded in the clinical trials. Despite scant real-world evidence of TKI-induced hepatotoxicity, a latest meta-analysis incorporating 43 studies claimed an overall incidence of $20 \%$ for all grade hepatotoxic events, which were in consistent with our results. ${ }^{8}$ Our findings demonstrated that hepatic toxicity related to EGFR-TKIs is well tolerable. A small proportion of patients developed severe hepatic events were discontinued with EGFR-TKI and retreated with decreased dose or switched to other types of EGFR-TKI after hepatoprotective 


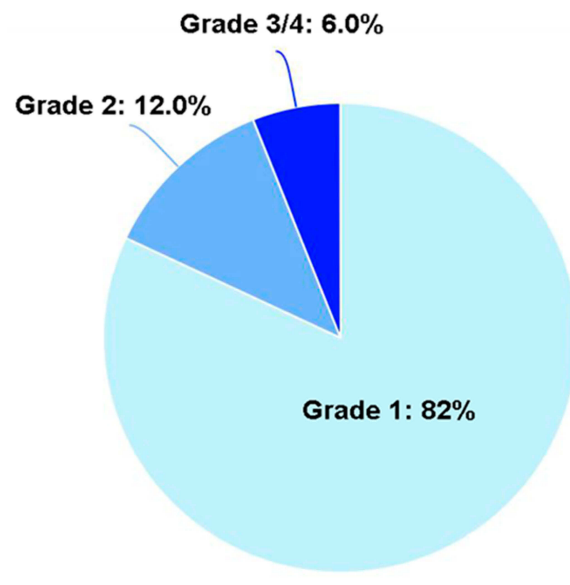

Gefitinib

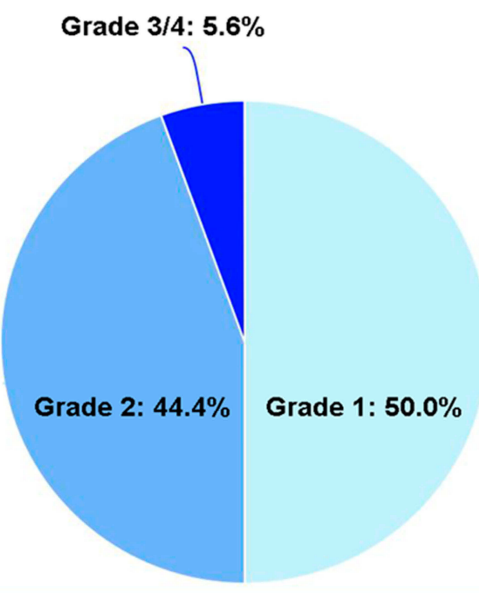

Erlotinib

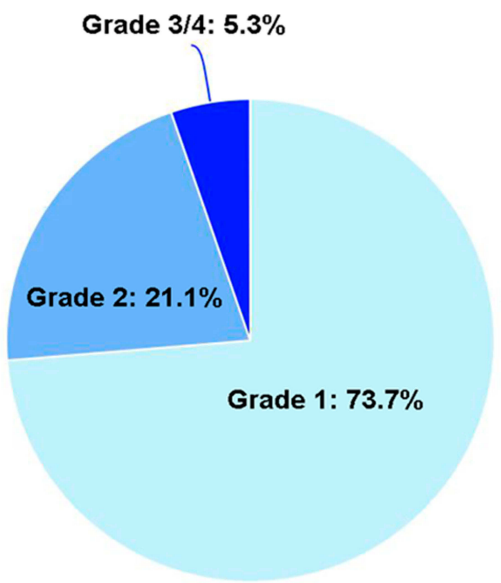

Icotinib

\begin{tabular}{llll}
\hline Grade & ALT, U/ & AST, U/ & TBil, $\mu \mathrm{mol} / 1$ \\
\hline $1(n=64)$ & $60(93.8 \%)$ & $15(23.4 \%)$ & $7(10.9 \%)$ \\
$2(n=18)$ & $17(94.4 \%)$ & $4(23.5 \%)$ & $1(5.6 \%)$ \\
$3 / 4(n=5)$ & $5(100 \%)$ & $3(60.0 \%)$ & $1(20.0 \%)$ \\
\hline
\end{tabular}

Figure I Characteristics of hepatotoxicity in patients receiving first-line EGFR-TKIs.

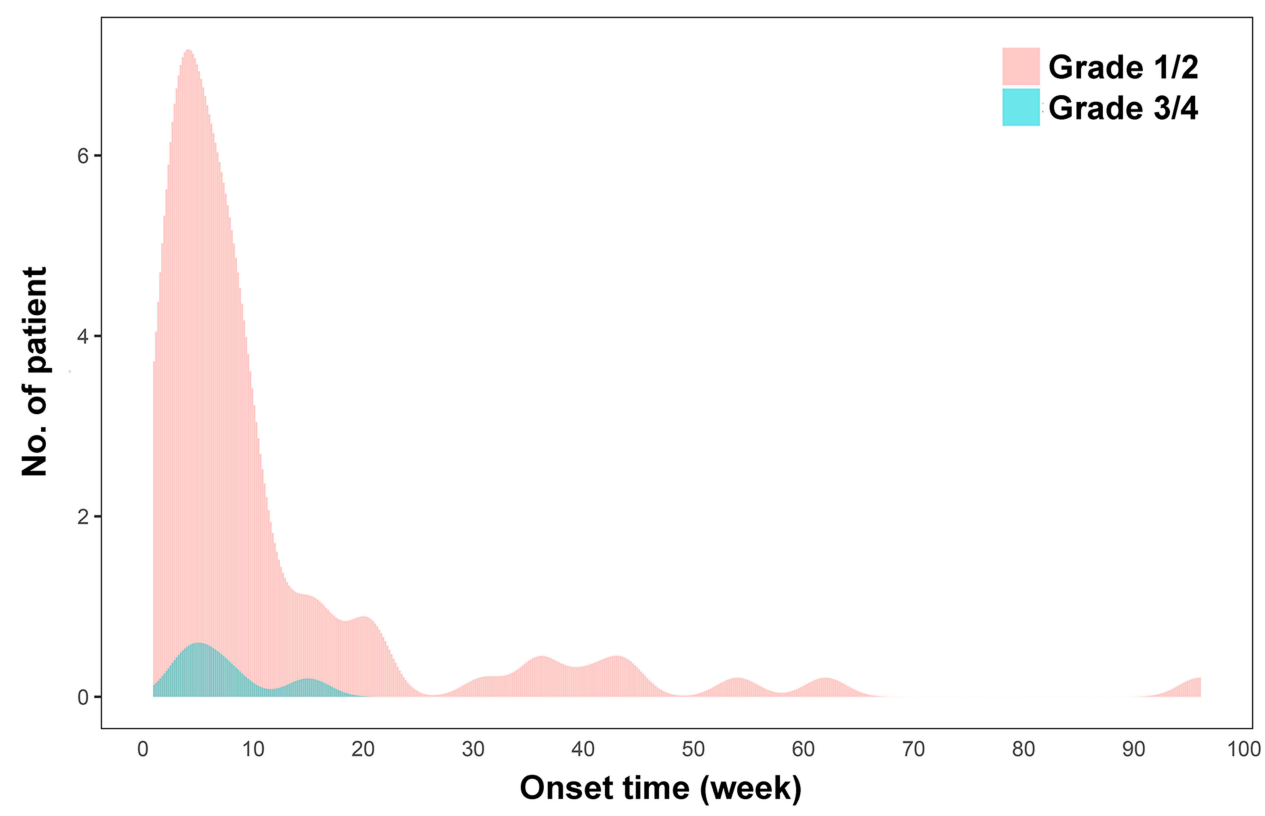

Figure 2 Onset time of EGFR-TKI-induced hepatotoxicity.

treatment. All patients recovered after the prompt intervention. Concerning the treatment outcome of this specific subgroup, current evidence suggests that TKI-induced hepatoxicity is not an on-target effect linked to its efficacy. ${ }^{9}$ A previous study based on a few cases of EGFR-TKI induced hepatotoxicity revealed that the approximate median progression-free survival (PFS) was 281 (range: 206-527) days which was consistent with the PFS results of first-generation EGFR-TKI in clinical trials on non-small cell lung cancer (NSCLC). ${ }^{10}$ 
Table 2 Logistic Regression Analysis for Hepatic Toxicity in Patients Undergoing First-Line EGFR-TKI Treatment

\begin{tabular}{|c|c|c|c|c|c|c|}
\hline \multirow[t]{2}{*}{ Variable } & \multicolumn{2}{|c|}{ Liver Dysfunction, No. (\%) } & \multicolumn{2}{|l|}{ Univariable Analysis } & \multicolumn{2}{|l|}{ Multivariable Analysis } \\
\hline & No & Yes & Odds Ratio (95\% Cl) & $P$ & Odds Ratio (95\% Cl) & $P$ \\
\hline \multicolumn{7}{|l|}{ Age } \\
\hline$<70$ years & $28 I(83.4)$ & $74(85.1)$ & Reference & & Reference & \\
\hline$\geq 70$ years & $56(16.6)$ & $13(14.9)$ & $0.882(0.458-1.698)$ & 0.706 & $0.986(0.425-2.284)$ & 0.973 \\
\hline \multicolumn{7}{|l|}{ Gender } \\
\hline Male & $147(43.6)$ & $38(43.7)$ & Reference & & Reference & \\
\hline Female & $190(56.4)$ & $49(56.3)$ & $0.998(0.620-1.605)$ & 0.992 & $1.515(0.736-3.119)$ & 0.260 \\
\hline \multicolumn{7}{|l|}{ Smoking Status } \\
\hline Never & $252(74.8)$ & $61(70.1)$ & Reference & & Reference & \\
\hline Ever & $85(25.2)$ & $26(29.9)$ & $1.264(0.75 \mathrm{I}-2.127)$ & 0.378 & $1.600(0.719-3.563)$ & 0.250 \\
\hline \multicolumn{7}{|l|}{ Drinking History } \\
\hline No & 331 (98.2) & 85 (97.7) & Reference & & Reference & \\
\hline Yes & $6(1.8)$ & $2(2.3)$ & $1.298(0.257-6.546)$ & 0.752 & $0.843(0.150-4.729)$ & 0.846 \\
\hline \multicolumn{7}{|l|}{$\mathrm{HBsAg}$ or $\mathrm{HCV}-\mathrm{Ab}$} \\
\hline Absent & $320(95.0)$ & $76(87.4)$ & Reference & & Reference & \\
\hline Present & $17(5.0)$ & II (I2.6) & $2.724(1.226-6.055)$ & 0.014 & $2.593(1.090-6.170)$ & 0.031 \\
\hline \multicolumn{7}{|l|}{ Pretreatment Liver Impairment } \\
\hline No & $312(92.6)$ & $67(77.0)$ & Reference & & Reference & \\
\hline Yes & $25(7.4)$ & $20(23.0)$ & 3.725 (1.956-7.097) & 0.000 & $3.460(1.746-6.855)$ & $<0.001$ \\
\hline \multicolumn{7}{|l|}{ ECOG PS } \\
\hline $0 \sim 1$ & $304(90.2)$ & $80(92.0)$ & Reference & & Reference & \\
\hline 2 & $33(9.8)$ & $7(8.0)$ & $0.806(0.344-1.890)$ & 0.620 & $0.881(0.296-2.619)$ & 0.820 \\
\hline Stage & & & & 0.148 & & 0.145 \\
\hline IIIB & $29(8.6)$ & $10(11.5)$ & Reference & & Reference & \\
\hline IV & $272(80.7)$ & $62(71.3)$ & $0.66 I(0.306-1.427)$ & 0.292 & $0.657(0.289-1.492)$ & 0.315 \\
\hline Recurrent & $36(10.7)$ & $15(17.2)$ & $1.208(0.473-3.086)$ & 0.692 & $1.268(0.467-3.443)$ & $0.64 I$ \\
\hline \multicolumn{7}{|l|}{ Liver Metastasis } \\
\hline No & $324(96.1)$ & $81(93.1)$ & Reference & & Reference & \\
\hline Yes & $13(3.9)$ & $6(6.9)$ & $\mathrm{I} .846(0.68 \mathrm{I}-5.006)$ & 0.228 & $2.397(0.829-6.932)$ & 0.107 \\
\hline
\end{tabular}

Abbreviations: $\mathrm{HBsAg}$, hepatitis B virus surface antigen; $\mathrm{HCV}-\mathrm{Ab}$, hepatitis C virus antibody; ECOG PS, Eastern Cooperative Oncology Group performance score.

Toxicity profiles are different for EGFR-TKIs harboring unique chemical structures. Although there have been no prospective trials comparing toxicity among different firstline EGFR-TKIs, a meta-analysis and a pooled analysis revealed that gefitinib was associated with significantly higher risks of elevated liver enzymes than erlotinib. ${ }^{7,11}$ However, another network meta-analysis did not demonstrate the similar results. ${ }^{8}$ By adjusting for potential affecting covariance, we confirmed that gefitinib and erlotinib did not differ in hepatic toxicity. Icotinib is the first homegrown EGFR-TKI approved by the Chinese Food and Drug Administration (CFDA) for the treatment of NSCLC. Because of its shorter half-life and a wider therapeutic window, icotinib has decreased the risk of drug-related adverse events compared with gefitinib or erlotinib. ${ }^{12-14}$ The percentage of icotinib-related elevated ALT/AST events was $6.8-8.1 \%$ in the Phase III trial ${ }^{15}$ and only $3.8 \%$ based on a meta-analysis including 15 studies of NSCLC populations. Similar results were found in this study that icotinib-related abnormal liver function appeared in $12.7 \%$ of patients, significantly less than that of gefitinib or erlotinib. Despite its structural superiority, distinctive metabolite characteristics of icotinib, especially the effect of metabolites M20 and M23 on liver toxic reactions, partly explain the lower risk of liver impairment. ${ }^{16}$

The onset of hepatic toxicity induced by TKIs is usually within the first 2 months of initiating treatment but may vary 
Table 3 Multivariate Logistic Analysis Comparing Hepatotoxicity Among Different EGFR-TKI Agent

\begin{tabular}{|l|l|l|l|l|}
\hline \multirow{2}{*}{ Variable } & \multicolumn{3}{|l|}{$\begin{array}{l}\text { Liver Dysfunction, No. } \\
\text { (\%) }\end{array}$} & \multicolumn{2}{l|}{$\begin{array}{l}\text { Multivariable } \\
\text { Analysis }\end{array}$} \\
\cline { 2 - 5 } & No & Yes & $\begin{array}{l}\text { Odds Ratio } \\
(95 \% \text { Cl) }\end{array}$ & $P$ \\
\hline Icotinib & $131(38.9)$ & $19(21.8)$ & & \\
\hline Gefitinib & $167(49.6)$ & $50(57.5)$ & & \\
\hline Erlotinib & $39(11.6)$ & $18(20.7)$ & & 0.119 \\
\hline Erlotinib vs Gefitinib & & & $\begin{array}{l}1.693 \\
(0.874-3.285)\end{array}$ & \\
\hline Gefitinib vs Icotinib & & & $\begin{array}{l}1.872 \\
(1.028-3.412)\end{array}$ & 0.040 \\
\hline Erlotinib vs Icotinib & & & 3.578 & 0.001 \\
& & & $(1.683-7.609)$ & \\
\hline
\end{tabular}

depending on the discrete spectrum of clinical characteristics and diseases. ${ }^{9}$ In this study, the median onset time of hepatotoxicity was 7 weeks, implying that a stringent monitoring of hepatic function should be performed within the first 2 months after TKI therapy. However, there was still a small proportion ( $\mathrm{n}=9,10.3 \%$ ) of patients developed liver dysfunction after half a year since the initiation of TKI, reminding us that a long observation of TKI-induced side effect is necessary although all these patients were of mild grade hepatotoxicity.

The mechanism underlying EGFR-TKI-induced hepatotoxicity has not yet been well clarified. ${ }^{17}$ Much of the available histological information suggested that hepatocellular necrosis was accounted for the most frequent form of TKI-induced liver injury. ${ }^{9}$ Our findings confirmed that increased aminotransferases were the most-seen pattern of liver injury. Approximately $10 \%$ of patients experienced elevated TBil, indicating a possibility of bile duct epithelial injury and cholestasis.

Pre-existing diseases such as viral infection or prior liver damage are related to hepatic toxicity arising during TKI therapy. ${ }^{18,19}$ Although TKIs are not generally associated with hepatitis reactivation, HBV reactivation after the use of small-molecule TKIs has been reported previously. ${ }^{20}$ In this study, hepatitis reactivation was not observed. However, treatment with antiviral agents could be considered in consultation with specialists in hepatology.

There are several limitations to our study. First, concomitant treatment such as herbal medicine that may affect liver function was not included in the analysis. Previous studies demonstrated that polypharmacy might cloud the picture of hepatotoxicity. ${ }^{21}$ Second, for patients with hepatitis virus infection, we were unable to consider patients with concurrent virus copy test with the intent to describe more dangerous conditions. In addition, we did not separately study the risk of hepatitis $B$ and $C$ because of the limited sample size of hepatitis $\mathrm{C}$ infection. Third, the current study had a limited sample size of patients developing hepatic injury; we, therefore, did not separately analyze those with more severe hepatotoxicity ( $\geq$ grade 2 ) that may provide more clinical significance since grade 1 hepatic injury was not generally considered to be significant in clinical practice.

In conclusion, first-generation EGFR-TKI-related hepatic events are well-tolerable. The different toxic profile of EGFRTKIs should be taken into account in the choice of treatment based on the patients' comorbidity and drug availability.

\section{Ethics and Consent Statement}

All procedures performed in studies involving human participants were in accordance with the ethical standards of the institutional and/or national research committee and with the 1964 Helsinki Declaration and its later amendments or comparable ethical standards.

\section{Funding}

This research did not receive any specific grant from funding agencies in the public, commercial, or not-forprofit sectors.

\section{Disclosure}

The authors have no conflicts of interest to declare.

\section{References}

1. Goldstraw P, Ball D, Jett JR, et al. Non-small-cell lung cancer. Lancet. 2011;378(9804):1727-1740. doi:10.1016/S0140-6736(10)62101-0

2. Teo YL, Ho HK, Chan A. Risk of tyrosine kinase inhibitors-induced hepatotoxicity in cancer patients: a meta-analysis. Cancer Treat Rev. 2013;39(2):199-206. doi:10.1016/j.ctrv.2012.09.004

3. Wu YL, Zhou C, Hu CP, et al. Afatinib versus cisplatin plus gemcitabine for first-line treatment of Asian patients with advanced non-small-cell lung cancer harbouring EGFR mutations (LUX-lung 6): an open-label, randomised Phase 3 trial. Lancet Oncol. 2014;15(2):213-222. doi:10.1016/S1470-2045(13)70604-1

4. Park K, Tan EH, O'Byrne K, et al. Afatinib versus gefitinib as first-line treatment of patients with EGFR mutation-positive non-small-cell lung cancer (LUX-Lung 7): a phase 2B, open-label, randomised controlled trial. Lancet Oncol. 2016;17(5):577-589. doi:10.1016/S1470-2045(16) 30033-X

5. Mitsudomi T, Morita S, Yatabe Y, et al. Gefitinib versus cisplatin plus docetaxel in patients with non-small-cell lung cancer harbouring mutations of the epidermal growth factor receptor (WJTOG3405): an open label, randomised phase 3 trial. Lancet Oncol. 2010;11(2):121-128. doi:10.1016/S1470-2045(09)70364-X 
6. Wu YL, Zhou C, Liam CK, et al. First-line erlotinib versus gemcitabine/cisplatin in patients with advanced EGFR mutation-positive non-small-cell lung cancer: analyses from the Phase III, randomized, open-label, ENSURE study. Ann Oncol. 2015;26(9):1883-1889. doi:10.1093/annonc/mdv270

7. Takeda M, Okamoto I, Nakagawa K. Pooled safety analysis of EGFR-TKI treatment for EGFR mutation-positive non-small cell lung cancer. Lung Cancer. 2015;88(1):74-79. doi:10.1016/j. lungcan.2015.01.026

8. Liu Y, Zhang Y, Feng G, et al. Comparison of effectiveness and adverse effects of gefitinib, erlotinib and icotinib among patients with non-small cell lung cancer: A network meta-analysis. Exp Ther Med. 2017;14(5):4017-4032. doi:10.3892/etm.2017.5094

9. Shah RR, Morganroth J, Shah DR. Hepatotoxicity of tyrosine kinase inhibitors: clinical and regulatory perspectives. Drug Saf. 2013;36 (7):491-503. doi:10.1007/s40264-013-0048-4

10. Yano Y, Namba Y, Mori M, et al. Treatment of non-small-cell lung cancer with erlotinib following gefitinib-induced hepatotoxicity: review of 8 clinical cases. Lung Cancer Int. 2012;2012:354657. doi: $10.1155 / 2012 / 354657$

11. Ding PN, Lord SJ, Gebski V, et al. Risk of treatment-related toxicities from EGFR tyrosine kinase inhibitors: a meta-analysis of clinical trials of gefitinib, erlotinib, and afatinib in advanced EGFR-mutated non-small cell lung cancer. J Thorac Oncol. 2017;12(4):633-643. doi:10.1016/j.jtho.2016.11.2236

12. Liu D, Jiang J, Zhang L, et al. Clinical pharmacokinetics of icotinib, an anti-cancer drug: evaluation of dose proportionality, food effect, and tolerability in healthy subjects. Cancer Chemother Pharmacol. 2014;73(4):721-727. doi:10.1007/s00280-014-2398-8

13. Shi Y, Zhang L, Liu X, et al. Icotinib versus gefitinib in previously treated advanced non-small-cell lung cancer (ICOGEN): a randomised, double-blind phase 3 non-inferiority trial. Lancet Oncol. 2013;14(10):953-961. doi:10.1016/S1470-2045(13)70355-3
14. Lee CC, Shiao HY, Wang WC, Hsieh HP. Small-molecule EGFR tyrosine kinase inhibitors for the treatment of cancer. Expert Opin Investig Drugs. 2014;23(10):1333-1348. doi:10.1517/13543784. 2014.928283

15. Shi YK, Wang L, Han BH, et al. First-line icotinib versus cisplatin/ pemetrexed plus pemetrexed maintenance therapy for patients with advanced EGFR mutation-positive lung adenocarcinoma (CONVINCE): a phase 3, open-label, randomized study. Ann Oncol. 2017;28(10):2443-2450. doi:10.1093/annonc/mdx359

16. Chen J, Zheng X, Liu DY, et al. Therapeutic effects and adverse drug reactions are affected by icotinib exposure and CYP2C19 and EGFR genotypes in Chinese non-small cell lung cancer patients. Asian Pac J Cancer Prev. 2014;15(17):7195-7200. doi:10.7314/APJCP.2014. 15.17.7195

17. Paech F, Bouitbir J, Krahenbuhl S. Hepatocellular toxicity associated with tyrosine kinase inhibitors: mitochondrial damage and inhibition of glycolysis. Front Pharmacol. 2017;8:367. doi:10.3389/ fphar.2017.00367

18. Bahirwani R, Reddy KR. Drug-induced liver injury due to cancer chemotherapeutic agents. Semin Liver Dis. 2014;34(02):162-171. doi:10.1055/s-0034-1375957

19. Ruofan H, Qiong Z, Xinli Z, Zhaohui C, Jingwei J, Xiaohua L. Relationships between hepatitis B infection status and liver dysfunction after chemotherapy of lung cancer patients in mainland China. Support Care Cancer. 2013;21(7):1821-1826. doi:10.1007/s00520013-1738-2

20. Bui N, Wong-Sefidan I. Reactivation of hepatitis B virus after withdrawal of erlotinib. Curr Oncol. 2015;22(6):430-432. doi:10.3747/ co.22.2665

21. Floyd J, Mirza I, Sachs B, Perry MC. Hepatotoxicity of chemotherapy. Semin Oncol. 2006;33(1):50-67. doi:10.1053/j. seminoncol.2005.11.002
Cancer Management and Research

\section{Publish your work in this journal}

Cancer Management and Research is an international, peer-reviewed open access journal focusing on cancer research and the optimal use of preventative and integrated treatment interventions to achieve improved outcomes, enhanced survival and quality of life for the cancer patient.

\section{Dovepress}

The manuscript management system is completely online and includes a very quick and fair peer-review system, which is all easy to use. Visit http://www.dovepress.com/testimonials.php to read real quotes from published authors. 